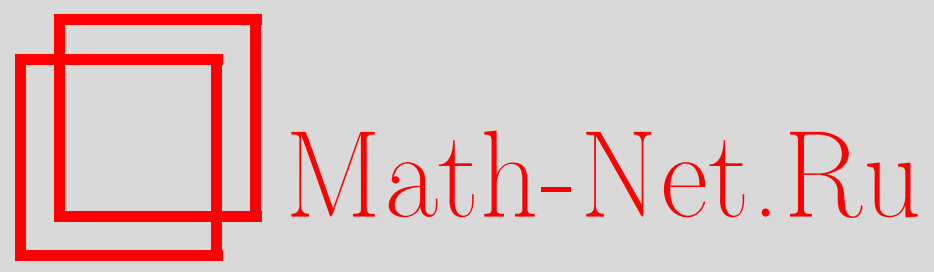

А. С. Рыбаков, Кратчайшие векторы решеток, связанных с линейным конгруэнтным генератором, Дискрет. матем., 2004, том 16, выпуск 4, 88-109

DOI: https://doi.org/10.4213/dm178

Использование Общероссийского математического портала Math-Net.Ru подразумевает, что вы прочитали и согласны с пользовательским соглашением http://www.mathnet.ru/rus/agreement

Параметры загрузки:

IP : 3.85 .5 .30

26 апреля 2023 г., 15:22:46

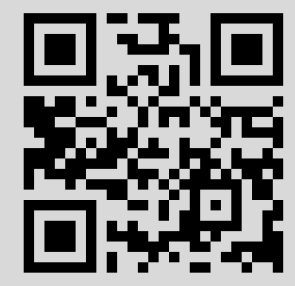




\title{
Кратчайшие векторы решеток, связанных с линейным конгруэнтным генератором
}

\author{
() 2004 г. А. С. Рыбаков
}

Пусть $\varepsilon>0-$ фиксированное число, $\mathscr{E} \subset \mathbf{R}^{s}-$ полная решетка определителя $\Delta \in \mathbf{Q}$. Назовем ее $\varepsilon$-регулярной, если $\lambda_{1}(\mathscr{E})>\Delta^{1 / s}(h(\Delta))^{-\varepsilon}$, где $\lambda_{1}(\mathscr{E})-$ длина ненулевого кратчайшего вектора решетки $\mathscr{E}$, а $h(\Delta)$ - максимум абсолютных величин числителя и знаменателя несократимой дроби для $\Delta$. В нашей работе рассматриваются две полные рсшетки пространства $\mathbf{R}^{s}$ :

- решетка $\mathscr{L}(a, W)$, связанная с линейной конгруэнтной последовательностью

$$
\left(x_{N}\right), \quad x_{N+1}=a x_{N} \quad(\bmod W), \quad N=1,2, \ldots,
$$

- двойственная к ней решетка $\mathscr{L}^{*}(a, W)$.

Существует гипотеза, утверждающая, что при любом натуральном $s$, любом $0<\varepsilon<\varepsilon_{0}(s)$ и любом $W>W_{0}(s, \varepsilon)$ все решетки $\mathscr{L}(a, W)$ и $\mathscr{L}^{*}(a, W)$ при $a=0,1, \ldots, W-1$ являются $\varepsilon$-регулярными за исключением множества чисел $a$ мощности, не большей $W^{1-\varepsilon}$.

В работе 1988 г. А. Фризом, Й. Хастадом, К. Каннаном, Дж. Лагариасом и А. Шамиром было доказано чуть более слабое утверждение в случае $s=3$ (число исключительных $a$ в их оценке не превосходит $W^{1-\varepsilon / 2}$ ). Кроме того, с помощью рассуждений указанной работы легко проверить справедливость гипотезы в случаях $s=1$ и $s=2$.

В нашей работе доказывается справедливость сформулированной гипотезы в случае $s=4$. Кроме того, с помощью рассуждений нашей работы можно слегка уточнить результат упомянутой выше работы, установив справедливость гипотезы в случае $s=3$.

Все указанные утверждения имеют приложения в теории восстановления линейной конгруэнтной последовательности (1) по старшим разрядам ее нескольких первых элементов.

\section{1. Введение}

Пусть $\mathscr{E}$ - полная решетка в пространстве $\mathbf{R}^{s}$ с фиксированным скалярным произведением $\langle\cdot, \cdot\rangle$, то есть множество линейных комбинаций с целыми коэффициентами заданной системы $s$ линейно независимых над $\mathbf{R}$ векторов, которые называются базисом решетки (см. гл. 2, §3, п. 2 в [1]).

Определителем $\mathscr{E}$ мы будем называть абсолютную величину определителя матрицы коэффициентов базиса $\mathscr{E}$ в произвольном ортонормированном базисе. Определитель решетки не зависит ни от выбора ортонормированного базиса, ни от выбора базиса решетки. 
Через $\lambda_{i}(\mathscr{E})$ обозначим $i$-й последовательный минимум решетки $\mathscr{E}$, то есть такое наименьшее число $R \in \mathbf{R}_{>0}$, что замкнутый шар радиуса $R$ с центром в нуле содержит $i$ линейно независимых векторов решетки $\mathscr{E}$.

Определение 1. Пусть $\varepsilon>0-$ фиксированное число, $\mathscr{E} \subset \mathbf{R}^{s}-$ полная решетка определителя $\Delta \in \mathbf{Q}$. Назовем ее $\varepsilon$-регулярной, если $\lambda_{1}(\mathscr{E})>\Delta^{1 / s}(h(\Delta))^{-\varepsilon}$, где $h(\Delta)-$ высота числа $\Delta$, равная по определению максимуму абсолютных величин числителя и знаменателя несократимой дроби для $\Delta$.

Замечание 1. Из теоремы Минковского о последовательных минимумах (см. теорему 5 гл. 8 в [2]) вытекает, что если полная решетка $\mathscr{E} \subset \mathbf{R}^{s}$ определителя $\Delta \in \mathbf{Q}$ является $\varepsilon$-регулярной, то имеет место неравенство

$$
\lambda_{s}(\mathscr{E})<\frac{2^{s}}{\gamma_{s}} \Delta^{1 / s}(h(\Delta))^{(s-1) \varepsilon},
$$

где

$$
\gamma_{s}=\frac{\pi^{s / 2}}{\Gamma(1+s / 2)}
$$

- объем единичного шара в $\mathbf{R}^{s}$. Следовательно, если число $\varepsilon$ достаточно мало, то все числа $\lambda_{i}(\mathscr{E})$ представляют собой величины приблизительно одного порядка.

Заметим, что существует определенная связь между $\varepsilon$-регулярностью решетки $\mathscr{E}$ и двойственной к ней решетки $\mathscr{E} *$, задаваемой равенством

$$
\mathscr{E}^{*}=\left\{y \in \mathbf{R}^{s}:\langle y, x\rangle \in \mathbf{Z} \text { для всех } x \in \mathscr{E}\right\} .
$$

Например, если решетка $\mathscr{E}^{*}$ является $\varepsilon$-регулярной и $\Delta-$ определитель $\mathscr{E}$, то число $\Delta^{-1}$ будет определителем $\mathscr{E}^{*}$ и мы получим, что $\lambda_{1}\left(\mathscr{E}^{*}\right) \geqslant \Delta^{-1 / s}(h(\Delta))^{-\varepsilon}$. Используя неравенство (см., например, [6])

$$
\lambda_{s}(\mathscr{E}) \lambda_{1}\left(\mathscr{E}^{*}\right) \leqslant s^{2},
$$

отсюда можно получить, что $\lambda_{s}(\mathscr{E}) \leqslant s^{2} \Delta^{1 / s}(h(\Delta))^{\varepsilon}$, а тогда по теореме Минковского о последовательных минимумах найдем, что

$$
\lambda_{1}(\mathscr{E}) \geqslant \frac{2^{s}}{s ! s^{2(s-1)} \gamma_{s}} \Delta^{1 / s}(h(\Delta))^{-\varepsilon(s-1)} .
$$

Следовательно, если число $\varepsilon$ мало, а число $h(\Delta)$ достаточно велико, то решетка $\mathscr{E}$ будет $\varepsilon_{0}$-регулярна для некоторого малого числа $\varepsilon_{0}$.

Пусть $s, W$ - натуральные числа, $a$ - целое число. Основным объектом исследования в данной работе будут следующие две полные решетки пространства $\mathbf{R}^{s}$ :

(1) решетка $\mathscr{L}(a, W)$, базисные векторы которой совпадают со строками матрицы

$$
\left(\begin{array}{ccccc}
W & 0 & 0 & \ldots & 0 \\
a & -1 & 0 & \ldots & 0 \\
a^{2} & 0 & -1 & \ldots & 0 \\
\ldots \ldots & \ldots & \ldots & \ldots & \ldots \\
a^{s-1} & 0 & 0 & \ldots & -1
\end{array}\right)
$$


(2) двойственная к ней решетка $\mathscr{L}^{*}(a, W)$, базисные векторы которой совпадают со строками матрищы

$$
\left(\begin{array}{ccccc}
1 / W & a / W & a^{2} / W & \ldots & a^{s-1} / W \\
0 & 1 & 0 & \ldots & 0 \\
0 & 0 & 1 & \ldots & 0 \\
\ldots \ldots \ldots \ldots \ldots \ldots \ldots \ldots \ldots \ldots \ldots \ldots & \ldots \ldots
\end{array}\right)
$$

Далее на протяжении всей работы размерность пространства $s$ будет считаться фиксированной, а числа $W$ и $a$ будут пробегать некоторые подмножества неотрицательных целых чисел. Легко видеть, что решетки $\mathscr{L}(a, W)$ и $\mathscr{L}^{*}(a, W)$ будут одни и те же для сравнимых между собой чисел $a$ по модулю $W$, поэтому, не ограничивая общности, можно считать, что $a \in I_{W}=\{0,1, \ldots, W-1\}$.

Решетки $\mathscr{L}(a, W)$ и $\mathscr{L}^{*}(a, W)$ рассматривались в 1988 г. в [6] в связи с исследованием задачи восстановления линейной рекуррентной последовательности

$$
x_{1}, x_{2}, x_{3}, \ldots, \quad x_{N} \in \mathbf{Z}, \quad 0 \leqslant x_{N}<W, \quad x_{N+1}=a x_{N} \quad(\bmod W),
$$

при известных $a, W$ в случае, если заданы $l$ старших двоичных разрядов каждого из чисел $x_{i}, i=1, \ldots, s$, где $l$ - некоторый параметр, зависящий от $a, W$ и $s$.

В [6] были доказаны две следующие теоремы.

Теорема 1 ([6]). Пусть число $W$ пробегает множество натуральных чисел, не делящихся на квадрат простого числа. Тогда при любом $0<\varepsilon<1 / s u W>W_{0}(s, \varepsilon)$ все решетки $\mathscr{L}(a, W)$ и $\mathscr{L}^{*}(a, W)$ при $a \in I_{W}$ будут в-регулярны за исключением множества чисел а мощности, не большей $W^{1-\varepsilon}$.

Теорема 2 ([6]). Пусть $s=3$, а число $W$ пробегает множество всех натуральных чисел. Тогда при любом $0<\varepsilon<1 / 3$ и любом $W>W_{0}(s, \varepsilon)$ все решетки $\mathscr{L}(a, W)$ и $\mathscr{L}^{*}(a, W)$ при $a \in I_{W}$ будут в-регулярны за исключением множества чисел а мощности, не больмей $W^{1-\varepsilon / 2}$.

Замечание 2. В [6] доказывалась $\varepsilon$-регулярность решеток $\mathscr{L}^{*}(a, W)$ для почти всех $a$, но из доказательства теорем легко вытекает соответствующее утверждение для решеток $\mathscr{L}(a, W)$.

С помощью теорем 1 и 2 был установлен следующий основной результат.

Теорема 3 ([6]). Пусть число $W$ пробегает множество натуральных чисел, не делящихся на квадрат простого числа, либо $s=3 u \mathrm{~W}$ пробегает множество всех натуральных чисел. Тогда при любом $0<\varepsilon<1 / s$ и любом $W>W_{0}(s, \varepsilon)$ существует такое исключительное множество $E=E(W, s, \varepsilon) \subset I_{W}$ мощности, не больщей $W^{1-\varepsilon / 2}$, что при любом $a \in I_{W} \backslash E$ верно следующее. Числа $x_{1}, \ldots, x_{s}$ последовательности (2) однозначно определены при задании свочх $l=\left\lfloor(1 / s+\varepsilon) \log _{2} W\right\rfloor$ старших двоччных разрядов $и$ существует алгоритм нахождения этих чисел, имеющий полиномиальное относительно $\log _{2} W+s$ время работы.

Цель данной работы состоит в доказательстве аналога теоремы 2 для $s=4$. Основной результат нашей работы имеет следующий вид. 
Теорема 4. Пусть $s=4$, а число $W$ пробегает множество всех натуральных чисел. Тогда при любом $0<\varepsilon<1 / 48$ и любом $W>W_{0}(s, \varepsilon)$ все решетки $\mathscr{L}(a, W)$ и $\mathscr{L}^{*}(a, W)$ при $a \in I_{W}$ будут в-регулярны за исключением множества чисел а мочности, не больuей $W^{1-\varepsilon}$.

Помимо этого, интерес может представлять теорема 5, которую мы формулируем в разделе 3 , а также некоторые утверждения о числе корней многочлена над кольцом вычетов, формулируемые в разделе 2 .

Из теоремы 4 можно извлечь очевидное уточнение теоремы 3. Формулировку мы опускаем. Кроме того, используя те же аргументы, что приводятся ниже для доказательства теоремы 4 , несложно показать, что при $0<\varepsilon<1 / 18$ оценку $W^{1-\varepsilon / 2}$ для количества исключительных чисел теоремы 2 можно заменить на $W^{1-\varepsilon}$.

Отметим еще одну интересную аналогию между известной гипотезой К. Малера (см. замечание к главе 7 в [2]) и гипотезой об $\varepsilon$-регулярности решетки $\mathscr{L}^{*}(a, W)$ для почти всех $a$. Пусть $|x| \mathbf{z}$ - расстояние от числа $x \in \mathbf{R}$ до ближайшего целого. Гипотеза К. Малера утверждает, что при любом $\varepsilon>0$ множество чисел $x \in \mathbf{R}$, для которых неравенство

$$
\max _{j=1, \ldots, s}\left|q x^{j}\right| \mathbf{z} \leqslant q^{-1 / s-\varepsilon}
$$

имеет бесконечное число целых решений $q$, есть множество лебеговой меры 0 . Для доказательства гипотезы об $\varepsilon$-регулярности решетки $\mathscr{L}^{*}(a, W)$ для почти всех $a$ надо в свою очередь показать, что при любом достаточно малом $\varepsilon>0$ и любом $W>W_{0}(s, \varepsilon)$ множество тех чисел $a \in I_{W}$, для которых неравенство

$$
\max _{j=0, \ldots, s-1}\left|t a^{j} / W\right|_{\mathbf{z}} \leqslant W^{-1 / s-\varepsilon}
$$

имеет нетривиальное решение $t \in I_{W}$, есть множество мощности, не большей $W^{1-\varepsilon}$.

\section{2. Оценка числа корней многочлена над кольцом вычетов}

Следующие обозначения будут общими для всех утверждений этого раздела:

$p$ - фиксированное простое число,

$v_{p}(x)-p$-показатель числа $x \in \mathbf{Q}$, определяющийся следующим образом: $v_{p}(0)=\infty$, для целого числа $x \neq 0$ показатель $v_{p}(x)$ есть наибольшее целое $m$, для которого $p^{m} \mid x$, наконец, для рационального $x=a / b$ с целыми $a$ и $b$ показатель $v_{p}(x)$ равен разности $v_{p}(a)-v_{p}(b)$;

$\mathbf{Q}_{p}$ - поле $p$-адических чисел, то есть пополнение поля $\mathbf{Q}$ по метрике $|x|_{p}=p^{-v_{p}(x)}$, порожденной $p$-показателем $v_{p}$; эта метрика однозначно продолжается до метрики на алгебраическом замыкании поля $\mathbf{Q}_{p}$ и его пополнении (см. $\left.[3,1]\right)$; всюду ниже символ $|\cdot|_{p}$ рассматривается в этом более широком смысле;

$\mathbf{Z}_{p}$ - кольцо целых $p$-адических чисел, которое можно определить двумя способами, либо как замыкание кольца $\mathbf{Z}$ в $\mathbf{Q}_{p}$, либо как единичный шар $\left\{x \in \mathbf{Q}_{p}:|x|_{p} \leqslant 1\right\}$ (см. гл. $4, \S 1$, п. 4 в [1]); 
$K$ - конечное расширение поля $\mathbf{Q}_{p}$;

$\mathbf{Z}_{K}$ - целое замыкание кольца $\mathbf{Z}_{p}$ в $K$, которое совпадает с единичным шаром $\left\{x \in K:|x|_{p} \leqslant 1\right\}$ в $K$ (см. гл. $4, \S 1$, п. 4 в [1]);

$f(z)=a_{n} \prod_{i=1}^{n}\left(z-\alpha_{i}\right) \in \mathbf{Z}[z]-$ многочлен степени $n$, разлагающийся в $K$ на линейные множители;

$D_{f}=a_{n}^{2 n-2} \prod_{i<j}\left(\alpha_{i}-\alpha_{j}\right)^{2}-$ дискриминант $f$.

Доказательство следующей леммы можно найти, например, в [8], но на русском языке оно, по всей видимости, не опубликовано. В целях полноты мы предпочли включить это доказательство.

Лемма 1 (лемма 3.1 в [8]). Имеет место равенство

$$
\left|a_{n}\right|_{p} \prod_{i=1}^{n} \max \left(1,\left|\alpha_{i}\right|_{p}\right)=|d|_{p},
$$

где $d$ - наибольший общий делитель коэффичиентов многочлена $f$.

Доказательство. Пусть числа $\alpha_{1}, \ldots, \alpha_{n}$ занумерованы так, что $\left|\alpha_{1}\right|_{p} \leqslant \ldots \leqslant\left|\alpha_{n}\right|_{p}$ и $a_{i}$ - коэффициент $f(z)$ при степени $z^{i}$. По формулам Виета

$$
a_{n-i} / a_{n}=(-1)^{i} \sum_{1 \leqslant s_{1}<\ldots<s_{i} \leqslant n} \alpha_{s_{1}} \ldots \alpha_{s_{i}}, \quad i=1, \ldots, n .
$$

Пусть $j$ есть максимум множества $\left\{i:\left|\alpha_{i}\right|_{p} \leqslant 1\right\}$, если это множество непусто, и $j=0$, если оно пусто. Поскольку самым большим в метрике $|\cdot|_{p}$ из слагаемых сумм (2) будет слагаемое $\alpha_{j+1} \ldots \alpha_{n}$ суммы для $a_{j} / a_{n}$, в силу неархимедовости метрики $|\cdot|_{p}$ справедливы равенства

$$
\max \left\{\left|a_{n-i} / a_{n}\right|_{p}: i=0,1, \ldots, n\right\}=\left|a_{j} / a_{n}\right|_{p}=\left|\alpha_{j+1} \ldots \alpha_{n}\right|_{p}=\prod_{i=1}^{n} \max \left(1,\left|\alpha_{i}\right|_{p}\right),
$$

следовательно,

$$
|d|_{p}=\max \left\{\left|a_{i}\right|_{p}: i=0,1, \ldots, n\right\}=\left|a_{n}\right|_{p} \prod_{i=1}^{n} \max \left(1,\left|\alpha_{i}\right|_{p}\right),
$$

что и требовалось доказать.

Следствие 1. Если $u, u_{1}, \ldots, u_{n}-$ произвольные неотрицательные челье числа $и$ $u \geqslant \max \left(u_{1}, \ldots, u_{n}\right)$, то число $a_{n}^{u} \alpha_{1}^{u_{1}} \ldots \alpha_{n}^{u_{n}}$ принадлежат $\mathbf{Z}_{K}$.

Доказательство. Очевидно, что достаточно исследовать случай $u=1, u_{i} \in\{0,1\}$, $i=1, \ldots, n$, поскольку рассматриваемое в условии следствия число можно представить в виде произведения чисел, у которых $u$ и $u_{1}, \ldots, u_{n}$ имеют указанный вид. Так как

$$
\left|a_{n} \alpha_{1}^{u_{1}} \ldots \alpha_{n}^{u_{n}}\right|_{p} \leqslant\left|a_{n}\right|_{p} \prod_{i=1}^{n} \max \left(1,\left|\alpha_{i}\right|_{p}\right) \leqslant 1
$$


(второе неравенство справедливо в силу леммы 1), число $a_{n} \alpha_{1}^{u_{1}} \ldots \alpha_{n}^{u_{n}}$ принадлежит единичному шару в $K$, а значит, и $\mathbf{Z}_{K}$.

Идея доказательства следующих двух лемм, точнее говоря, идея о том, что для оценки снизу значения многочлена в любой точке $w$, являющейся его корнем по модулю $p^{e}$, следует рассмотреть разности $w-\alpha_{i}$ в поле $K$, навеяна леммой 1.7 книги [5], где аналогичная ситуация рассмотрена по отношению к обычной метрике модуля и конечному расширению поля $\mathbf{Q}$.

Лемма 2. Для любого $w \in K$ имеет место оченка

$$
|f(w)|_{p} \geqslant\left|w-\alpha_{s}\right|_{p}\left|f^{\prime}(w)\right|_{p},
$$

где $\alpha_{s}-$ ближайший $\kappa w$ корень многочлена $f$.

Доказательство. При любом $i=1, \ldots, n$ ввиду неравенства $\left|w-\alpha_{s}\right|_{p} \leqslant\left|w-\alpha_{i}\right|_{p}$ имеет место оценка

$$
\prod_{j: j \neq i}\left|w-\alpha_{j}\right|_{p} \leqslant \prod_{j: j \neq s}\left|w-\alpha_{j}\right|_{p}
$$

отсюда в силу неархимедовости метрики $|\cdot|_{p}$ следует оценка

$$
\left|f^{\prime}(w)\right|_{p}=\left|a_{n}\right|_{p}\left|\sum_{i=1}^{n} \prod_{j: j \neq i}\left(w-\alpha_{j}\right)\right|_{p} \leqslant\left|a_{n}\right|_{p} \prod_{j: j \neq s}\left|w-\alpha_{j}\right|_{p}=|f(w)|_{p} /\left|w-\alpha_{s}\right|_{p},
$$

что и требовалось доказать.

Лемма 3. Для любого $w \in K$ имеет место оценка

$$
|f(w)|_{p} \geqslant\left|w-\alpha_{s}\right|_{p} p^{-r / 2},
$$

где $s$ - то же, что и в лемме 2, а $r=\nu_{p}\left(D_{f}\right)$.

Доказательство. При $D_{f}=0$ утверждение очевидно. Пусть $D_{f} \neq 0$, то есть все корни $\alpha_{i}$ попарно различны. Будем для простоты считать, что $s=1$. Поскольку $\left|w-\alpha_{1}\right|_{p} \leqslant\left|w-\alpha_{j}\right|_{p}$ при любом $j$, то $\left|\alpha_{1}-\alpha_{j}\right|_{p}=\left|\alpha_{1}-w+w-\alpha_{j}\right|_{p} \leqslant\left|w-\alpha_{j}\right|_{p}$, отсюда

$$
|f(w)|_{p} \geqslant\left|w-\alpha_{1}\right|_{p}\left|a_{n}\right|_{p} \prod_{j \geqslant 2}\left|\alpha_{1}-\alpha_{j}\right|_{p} .
$$

Рассмотрим число

$$
\begin{aligned}
\sqrt{D_{f}} & =a_{n}^{n-1} \prod_{i<j}\left(\alpha_{i}-\alpha_{j}\right)=a_{n} \prod_{j \geqslant 2}\left(\alpha_{1}-\alpha_{j}\right) D^{\prime}, \\
D^{\prime} & =a_{n}^{n-2} \prod_{i \geqslant 2} \prod_{j>i}\left(\alpha_{i}-\alpha_{j}\right) .
\end{aligned}
$$

В силу следствия 1 леммы 1 число $D^{\prime}$ принадлежит $\mathbf{Z}_{K}$, следовательно, $\left|D^{\prime}\right|_{p} \leqslant 1$. Отсюда вытекает, что

$$
p^{-r / 2}=\left|\sqrt{D_{f}}\right|_{p} \leqslant\left|a_{n}\right|_{p} \prod_{j \geqslant 2}\left|\alpha_{1}-\alpha_{j}\right|_{p}
$$

следовательно, в силу (3) справедливо неравенство $|f(w)|_{p} \geqslant\left|w-\alpha_{1}\right|_{p} p^{-r / 2}$, что и требовалось доказать. 
Лемма 4. Число корней многочлена $f$ по модулю $p^{e}$ не превосходит $n p^{v}$, где

$$
v=\max \left\{v_{p}\left(f^{\prime}(x)\right): x \in \mathbf{Z}, f(x)=0 \quad\left(\bmod p^{e}\right)\right\} .
$$

Доказательство. При $v \geqslant e$ утверждение очевидно. Пусть $v \leqslant e-1$ и $w_{1}, \ldots, w_{N}-$ все корни $f$ по модулю $p^{e}$. Разобьем их на группы. В $i$-ю группу включаются те $w_{j}$, для которых ближайшим корнем $f$ в $K$ будет $\alpha_{i}$ (если два корня из $K$ одинаково близки к некоторому $w_{j}$, то число $w_{j}$ включается в любую из соответствующих двух групп). Допустим противное, то есть, что $N>n p^{v}$. Тогда одна из групп, например, первая, содержит больше $p^{v}$ элементов. Пусть для простоты $w_{1}, \ldots, w_{N_{0}}-$ все элементы первой группы, где $N_{0}>p^{\nu}$. Не ограничивая общности, можно считать, что

$$
0 \leqslant w_{1}<w_{2}<\ldots<w_{N_{0}}<p^{e} .
$$

По определению числа $v$ все числа $\left|f^{\prime}\left(w_{i}\right)\right|_{p}$ будут не меньше $p^{-v}$, отсюда по лемме 2

$$
\left|f\left(w_{i}\right)\right|_{p} \geqslant\left|\alpha_{1}-w_{i}\right|_{p} p^{-v}, \quad i=1, \ldots, N_{0} \text {. }
$$

Последнее означает, что

$$
\left|\alpha_{1}-w_{i}\right|_{p} \leqslant p^{-e+\nu}, \quad i=1, \ldots, N_{0},
$$

то есть, что

$$
\left|w_{i}-w_{j}\right|_{p} \leqslant p^{-e+v}, \quad i, j=1, \ldots, N_{0},
$$

то есть, что

$$
p^{e-v} \mid\left(w_{i}-w_{j}\right), \quad i, j=1, \ldots, N_{0} \text {. }
$$

Отсюда ввиду (4) вытекает оценка

$$
w_{N_{0}} \geqslant w_{1}+p^{e-v}\left(N_{0}-1\right) \geqslant w_{1}+p^{e-v}\left(p^{\nu}+1-1\right) \geqslant p^{e},
$$

противоречащая (4), что и доказывает лемму.

Следствие 2. Пусть v - фиксированное челое неотричательное число. Тогда число корней $w$ многочлена $f$ по модулю $p^{e}$, удовлетворяющих неравенству $v_{p}\left(f^{\prime}(w)\right) \leqslant v$, не превосходит $n p^{v}$.

Лемма 5. Число корней многочлена $f$ по модулю $p^{e}$ не превосходит $n p^{\lfloor r / 2\rfloor}$, где $r=v_{p}\left(D_{f}\right) u\lfloor\cdot\rfloor-$ челая часть снизу.

Доказательство. Доказательство аналогично доказательству леммы 4, только вместо оценки

$$
\left|f\left(w_{i}\right)\right|_{p} \geqslant\left|\alpha_{1}-w_{i}\right|_{p}\left|f^{\prime}\left(w_{i}\right)\right|_{p}, \quad i=1, \ldots, N_{0},
$$

леммы 2 мы пользуемся оценкой леммы 3

$$
\left.f\left(w_{i}\right)\right|_{p} \geqslant\left|\alpha_{1}-w_{i}\right|_{p} p^{-r / 2}, \quad i=1, \ldots, N_{0} .
$$


В предположении, что $N>n p^{\lfloor r / 2\rfloor}$, то есть, что $N_{0}>p^{\lfloor r / 2\rfloor}$, получаем оценки

$$
\left|w_{i}-w_{j}\right|_{p} \leqslant p^{-e+r / 2}, \quad i, j=1, \ldots, N_{0},
$$

то есть

$$
v_{p}\left(w_{i}-w_{j}\right) \geqslant e-r / 2, \quad i, j=1, \ldots, N_{0}
$$

Числа $v_{p}\left(w_{i}-w_{j}\right)$ целые, значит,

$$
v_{p}\left(w_{i}-w_{j}\right) \geqslant e-\lfloor r / 2\rfloor, \quad i, j=1, \ldots, N_{0} .
$$

Отсюда точно так же, как в лемме 3, вытекает противоречие с (4) и лемма тем самым доказана.

\section{3. Редукция}

Прежде чем доказывать теорему 4, нам будет удобно ввести несколько определений и обозначений.

Высотой многочлена $\varphi(x) \in \mathbf{C}[x]$ мы будем называть максимум модулей его коэффициентов. Многочлен $\varphi(x) \in \mathbf{Z}[x]$ назовем $W$-примитивным, если для любого простого числа $p \mid W$ найдется коэффициент $\varphi$, не делящийся на $p$. Символ $O$ во всех оценках ниже скрывает в себе абсолютную постоянную.

Далее, пусть $d(n)$ - число натуральных делителей натурального числа $n, \omega(n)$ - число простых делителей натурального числа $n$.

Имеют место оценки, которые ниже будут часто использоваться (см. теорему 5.2 главы 1 в [4] для величины $d(n)$ и формулу (5.5) в сочетании с теоремой 3.3 этой же главы для величины $\omega(n))$,

$$
d(n)=e^{O((\ln n) / \ln \ln n)}, \quad \omega(n)=O((\ln n) / \ln \ln n), \quad n \geqslant 3 .
$$

Наша ближайшая цель состоит в том, чтобы вывести сформулированную выше теорему 4 из следующей теоремы.

Теорема 5. Для любых достаточно большого натурального числа $W$ и вещественного $1 \leqslant R \leqslant W^{1 / 4}$ количество чисел а из множества $I_{W}=\{0,1, \ldots, W-1\}$, для которых сушествует многочлен $0 \not \equiv \varphi(x) \in \mathbf{Z}[x]$ степени, не больщей 3 , и высоты, не большей $R$, такой, что $\varphi(a)=0(\bmod W)$, не превосходит величины $W^{O(1 / \ln \ln W)} \max \left(R^{4}, W^{12 / 13} R^{2 / 13}\right)$.

Сначала оценим число $N$ решеток $\mathscr{L}(a, W), a \in I_{W}$, которые не являются $\varepsilon$-регулярными и для которых согласно определению число $а$ должно принадлежать множеству

$$
\begin{aligned}
\left\{a \in I_{W}: \text { существует многочлен } 0 \neq \varphi(x)=\sum_{j=0}^{3} \varphi_{j} x^{j} \in \mathbf{Z}[x]\right. \text { такой, что } \\
\qquad \sqrt{\left.\sum_{j=0}^{3} \varphi_{j}^{2} \leqslant W^{1 / 4-\varepsilon} \text { и } \varphi(a)=0 \quad(\bmod W)\right\}} .
\end{aligned}
$$


Возьмем $R=W^{1 / 4-\varepsilon}$, тогда по теореме 5

$$
N \leqslant W^{O(1 / \ln \ln W)} \max \left(R^{4}, W^{12 / 13} R^{2 / 13}\right) .
$$

Первый множитель правой части этого неравенства при достаточно большом $W$ не превосходит $W^{\varepsilon / 2}$, поэтому остается показать, что

$$
\frac{\varepsilon}{2}+\max \left(1-4 \varepsilon, \frac{12}{13}+\frac{2}{13}\left(\frac{1}{4}-\varepsilon\right)\right) \leqslant 1-\varepsilon
$$

или что

$$
13 \varepsilon+\max (26-104 \varepsilon, 25-4 \varepsilon) \leqslant 26-26 \varepsilon
$$

при $\varepsilon<1 / 48$. Последнее неравенство легко проверяется непосредственно.

Для числа $N^{*}$ решеток $\mathscr{L}^{*}(a, W), a \in I_{W}$, которые не являются $\varepsilon$-регулярными, рассуждение будет немного сложней. Заметим, что ввиду равенства

$$
W W^{-1 / 4-\varepsilon}=W^{3\left(1 / 4-\varepsilon_{0}\right)}, \quad \varepsilon_{0}=\varepsilon / 3,
$$

$\varepsilon$-регулярность решетки $\mathscr{L}^{*}(a, W)$ с определителем $W^{-1}$ равносильна $\varepsilon_{0}$-регулярности решетки $W \mathscr{L}^{*}(a, W)=\{W x: x \in \mathscr{L}(a, W)\}$ с определителем $W^{3}$, поэтому число $N^{*}$ равно также числу решеток $W \mathscr{L}^{*}(a, W)$, не являющихся $\varepsilon_{0}$-регулярными. Пусть $R=W^{1 / 4-\varepsilon_{0}}$. Свойство решетки $W \mathscr{L}^{*}(a, W)$ не быть $\varepsilon_{0}$-регулярной по определению означает, что число $a$ принадлежит множеству

$$
S_{R}=\left\{a \in I_{W}: \text { сушествует такое } t, 1 \leqslant t \in I_{W}, \text { что }\left|t a^{i}\right|_{W Z} \leqslant R^{3} \text { для } i=0,1,2,3\right\},
$$

где через $|x| w Z$ обозначено расстояние (в обычной метрике) от числа $x \in \mathbf{R}$ до множества $W \mathbf{Z}$ целых чисел, кратных числу $W$.

Следуя работе [6], рассмотрим при любом фиксированном натуральном $d \mid W$ множество

$$
S_{R, d}=\left\{a \in I_{W}: \text { существует такое } t, 1 \leqslant t \in I_{W},\right.
$$

что наибольший общий делитель $t$ и $W$ равен $d$ и $\left.\left|t a^{i}\right|_{W Z} \leqslant R^{3}, i=0, \ldots, 3\right\}$.

Тогда $S_{R}=\bigcup_{d \mid W} S_{R, d}$ и $d \leqslant R^{3}$ по определению множества $S_{R, d}$. Для оценки числа элементов множества $S_{R, d}$ мы используем лемму 3.2 работы [6], согласно которой при любом $a \in S_{R, d}$ существует многочлен $0 \not \equiv \varphi(x) \in \mathbf{Z}[x]$ степени, не большей 3 , и высоты, не большей $\left(8 R^{3} / d\right)^{1 / 3}=2 R / d^{1 / 3}$, такой, что $\varphi(a)=0(\bmod W / d)$. Применяя теорему 5 , получим, что число таких $a$ по модулю $W / d$ (то есть число классов вычетов $\left.\left\{a(\bmod W / d): a \in S_{R, d}\right\}\right)$ не превосходит величины

$$
W^{O(1 / \ln \ln W)} \max \left(\left(\frac{2 R}{d^{1 / 3}}\right)^{4},\left(\frac{W}{d}\right)^{12 / 13}\left(\frac{2 R}{d^{1 / 3}}\right)^{2 / 13}\right),
$$

соответственно, число таких $a$ по модулю $W$ для фиксированного $d$ не превосходит величины

$$
\begin{aligned}
d W^{O(1 / \ln \ln W)} \max \left(\left(\frac{2 R}{d^{1 / 3}}\right)^{4},\left(\frac{W}{d}\right)^{12 / 13}\left(\frac{2 R}{d^{1 / 3}}\right)^{2 / 13}\right) & \\
& =W^{O(1 / \ln \ln W)} \max \left(\frac{R^{4}}{d^{1 / 3}}, W^{12 / 13} R^{2 / 13} d^{1 / 39}\right)
\end{aligned}
$$


Так как $d \leqslant R^{3}$, число элементов множества $S_{R, d}$ не превосходит величины

$$
W^{O(1 / \ln \ln W)} \max \left(R^{4}, W^{12 / 13} R^{3 / 13}\right),
$$

поэтому число $N^{*}$ элементов множества $S_{R}$ не превосходит величины

$$
\begin{aligned}
\sum_{d \mid W} W^{O(1 / \ln \ln W)} \max \left(R^{4}, W^{12 / 13} R^{3 / 13}\right) & =d(W) W^{O(1 / \ln \ln W)} \max \left(R^{4}, W^{12 / 13} R^{3 / 13}\right) \\
& =W^{O(1 / \ln \ln W)} \max \left(R^{4}, W^{12 / 13} R^{3 / 13}\right) .
\end{aligned}
$$

По условию число $\delta=1 / 48-\varepsilon$ положительно, поэтому первый множитель в (5) при достаточно большом $W$ не будет превосходить $W^{\varepsilon \delta}$. Нам остается показать, что

$$
\varepsilon \delta+\max \left(1-4 \varepsilon_{0}, \frac{12}{13}+\frac{3}{13}\left(\frac{1}{4}-\varepsilon_{0}\right)\right) \leqslant 1-\varepsilon, \quad \varepsilon_{0}=\varepsilon / 3,
$$

или что

$$
\varepsilon \delta+\max \left(1-\frac{4 \varepsilon}{3}, \frac{12}{13}+\frac{3}{52}-\frac{\varepsilon}{13}\right) \leqslant 1-\varepsilon,
$$

или что

$$
156 \varepsilon \delta+\max (156-208 \varepsilon, 153-12 \varepsilon) \leqslant 156-156 \varepsilon,
$$

или что

$$
156 \varepsilon \delta+144 \varepsilon \leqslant 3
$$

Последнее неравенство легко проверить непосредственно.

Тем самым, мы показали, что теорема 4 вытекает из теоремы 5. Теперь покажем, что теорему 5 можно в свою очередь вывести из следующей теоремы.

Теорема 6. Для любых достаточно большого натурального числа $W$ и вещественного $R, 1 \leqslant R \leqslant W^{1 / 4}$, число тех а из множества $I_{W}=\{0,1, \ldots, W-1\}$, для которых существует $W$-примитивный многочлен $0 \not \equiv \varphi(x) \in \mathbf{Z}[x]$ степени, не большей 3, и высоты, не большей $R$, такой, что $\varphi(a)=0(\bmod W)$, не превосходит величины $W^{O(1 / \ln \ln W)} \max \left(R^{4}, W^{12 / 13} R^{2 / 13}\right)$.

Пусть $a \in I_{W}$ обладает тем свойством, что существует многочлен $0 \not \equiv \varphi(x) \in \mathbf{Z}[x]$ степени, не большей 3 , и высоты, не большей $R$, такой, что $\varphi(a)=0(\bmod W)$. Тогда при некотором натуральном $d \mid W, d \leqslant R$, число $a$ будет обладать тем свойством, что существует $W$-примитивный многочлен $0 \not \equiv \varphi(x) \in \mathbf{Z}[x]$ степени, не большей 3 , и высоты, не большей $R / d$, такой, что $\varphi(a)=0(\bmod W / d)$. По теореме 6 число таких $a$ по модулю $W / d$ не превосходит величины

$$
W^{O(1 / \ln \ln W)} \max \left(\left(\frac{R}{d}\right)^{4},\left(\frac{W}{d}\right)^{12 / 13}\left(\frac{R}{d}\right)^{2 / 13}\right),
$$

4 Дискретная математика, т. 16 №4 
соответственно число таких $a$ по модулю $W$ для фиксированного $d$ не превосходит величины

$$
d W^{O(1 / \ln \ln W)} \max \left(\left(\frac{R}{d}\right)^{4},\left(\frac{W}{d}\right)^{12 / 13}\left(\frac{R}{d}\right)^{2 / 13}\right) \leqslant W^{O(1 / \ln \ln W)} \max \left(R^{4}, W^{12 / 13} R^{2 / 13}\right) .
$$

Общее количество чисел $a$ теоремы 5 не будет превосходить величины

$$
\begin{aligned}
\sum_{d \mid W} W^{O(1 / \ln \ln W)} \max \left(R^{4}, W^{12 / 13} R^{2 / 13}\right) & =d(W) W^{O(1 / \ln \ln W)} \max \left(R^{4}, W^{12 / 13} R^{2 / 13}\right) \\
& \leqslant W^{O(1 / \ln \ln W)} \max \left(R^{4}, W^{12 / 13} R^{2 / 13}\right)
\end{aligned}
$$

что и требовалось доказать.

Таким образом, для доказательства теоремы 4 нам достаточно доказать теорему 6, чему будут посвящены следующие пять разделов.

\section{4. Отображение $\eta$}

Пусть $W=p_{1}^{F_{1}} \ldots p_{r}^{F_{r}}$, где $\dot{p}_{i}-$ простые числа, $F_{i} \in \mathbf{N}, i=1, \ldots, r,-$ каноническое разложение числа $W$. Пусть $I_{W}=\{0,1, \ldots, W-1\}$ и

$$
\begin{aligned}
& E(R, W)=\left\{a \in I_{W}: \varphi(a)=0 \quad(\bmod W) \text { для некоторого } W\right. \text {-примитивного } \\
& \text { многочлена } 0 \not \equiv(x) \in \mathbf{Z}[x] \text { степени, не большей } 3, \text { и высоты, не большей } R\} .
\end{aligned}
$$

Сопоставим произвольному элементу $a \in E(R, W)$ некоторое множество наборов $\eta(a)=\left\{\left(a_{0}, w, w^{\prime}, v, \varphi\right)\right\}$ следующим образом. Прежде всего возьмем произвольный $W$-примитивный ненулевой многочлен $\varphi$ с целыми коэффициентами степени, не большей 3 , и высоты, не большей $R$, такой, что $\varphi(a)=0(\bmod W)($ если существует несколько таких многочленов, то следующие ниже действия выполняются для каждого из них). Далее, пусть

$$
\begin{gathered}
f_{i}=\min \left(\left\lfloor F_{i} / 2\right\rfloor, v_{p_{i}}\left(\varphi^{\prime}(a)\right)\right), \quad i=1, \ldots, r \\
w=\prod_{i=1}^{r} p_{i}^{f_{i}}, \quad a_{0}=a \quad(\bmod w), \quad a_{0} \in I_{w}=\{0,1, \ldots, w-1\} .
\end{gathered}
$$

Нам остается определить числа $w^{\prime}$ и $v$. Заметим, что при любом $i=1, \ldots, r$ число $a_{0}$ удовлетворяет системе сравнений $\Sigma_{i}$

$$
\varphi\left(a_{0}\right)=0 \quad\left(\bmod p_{i}^{2 f_{i}}\right), \quad \varphi^{\prime}\left(a_{0}\right)=0 \quad\left(\bmod p_{i}^{f_{i}}\right),
$$

потому что этой системе удовлетворяет число $a$ (по определению) и этой же системе будет удовлетворять любое число, сравнимое с $a$ по модулю $p_{i}^{f_{i}}$ (в силу формулы Тейлора).

Положим $f_{i}^{\prime}=f_{i}+1$, если $2 \nmid F_{i}, f_{i}=\left\lfloor F_{i} / 2\right\rfloor>0$ и $a_{0}$ удовлетворяет расширенной системе $\Sigma_{i}^{\prime}$

$$
\varphi\left(a_{0}\right)=0 \quad\left(\bmod p_{i}^{2 f_{i}+1}\right), \quad \varphi^{\prime}\left(a_{0}\right)=0 \quad\left(\bmod p_{i}^{f_{i}+1}\right), \quad \varphi^{\prime \prime}\left(a_{0}\right) / 2=0 \quad\left(\bmod p_{i}\right) .
$$


В противном случае полагаем $f_{i}^{\prime}=f_{i}$. После этого возьмем

$$
w^{\prime}=\prod_{i=1}^{r} p_{i}^{f_{i}^{\prime}}, \quad v=\frac{w^{\prime}}{w} .
$$

Этим описание множества $\eta(a)$ для чисел $a \in E(R, W)$ завершается.

Лемма 6. Если прообраз $\eta^{-1}\left(a_{0}, w, w^{\prime}, v, \varphi\right)$ не пуст, то его мощность $N$ удовлетворяет неравенствам $w^{\prime} \leqslant N \leqslant 3^{r} w^{\prime}$, где $r$ - число простых, входящих в разложение $W$.

Доказательство. Пусть $\varnothing \neq E^{\prime}-$ рассматриваемый прообраз. По китайской теореме об остатках достаточно показать, что число $N_{0}$ элементов множества $\left\{a\left(\bmod p_{i}^{F_{i}}\right): a \in E^{\prime}\right\}$ удовлетворяет неравенствам $p_{i}^{f_{i}^{\prime}} \leqslant N_{0} \leqslant 3 p_{i}^{f_{i}^{\prime}}$. Для этого рассмотрим несколько случаев.

1. Пусть $f_{i}<\left\lfloor F_{i} / 2\right\rfloor$. Тогда $f_{i}^{\prime}=f_{i}$. Для доказательства нижней оценки $N_{0} \geqslant p_{i}^{f_{i}}$ заметим, что если $a \in E^{\prime}$, то для любого целого $t$, делящегося на $W_{i}^{\prime}=W / p_{i}^{F_{i}}$, наименьший неотрицательный вычет числа $a+p_{i}^{F_{i}-f_{i}} t$ по модулю $W$ также будет принадлежать множеству $E^{\prime}$.

Действительно, пусть $\tilde{a} \in I_{W}, \tilde{a}=a+p_{i}^{F_{i}-f_{i}} t(\bmod W)$. Тогда из формулы Тейлора и определения числа $f_{i}$ следует, что $\varphi(\tilde{a})=0(\bmod W)$. Таким образом, $\tilde{a} \in E(R, W)$. Далее, из тех же соображений легко вытекает, что и остальные компоненты в наборе $\left(\tilde{a}_{0}, \tilde{w}, \tilde{w}^{\prime}, \tilde{v}, \varphi\right) \in \eta(\tilde{a})$ будут теми же, что и в наборе $\left(a_{0}, w, w^{\prime}, v, \varphi\right)$. Тем самым наше утверждение доказано.

Возьмем теперь систему из $p_{i}^{f_{i}}$ чисел $t$, сравнимых с 0 по модулю $W_{i}^{\prime}$ и сравнимых соответственно с числами $0,1, \ldots, p_{i}^{f_{i}}-1$ по модулю $p_{i}^{F_{i}}$. Тогда соответствующая система наименьших неотрицательных вычетов чисел $a+p_{i}^{F_{i}-f_{i}} t$ по модулю $W$ для таких $t$ будет состоять точно из $p_{i}^{f_{i}}$ элементов, несравнимых друг с другом по модулю $p_{i}^{F_{i}}$, следовательно, $N_{0} \geqslant p_{i}^{f_{i}}$.

Оценка сверху для $N_{0}$ имеет место в силу следствия 2 леммы 4.

2. Пусть $f_{i}=\left\lfloor F_{i} / 2\right\rfloor$ и $2 \mid F_{i}$. Тогда снова $f_{i}^{\prime}=f_{i}$, так что оценка снизу для $N_{0}$ сразу вытекает из рассуждений предыдущего пункта. Оценка сверху вытекает из того, что всякое $a \in E^{\prime}$ должно быть по определению сравнимо с $a_{0}$ по модулю $p_{i}^{f_{i}}$, а число таких попарно несравнимых друг с другом вычетов по модулю $p_{i}^{F_{i}}$ равно $p_{i}^{F_{i}-f_{i}}=p_{i}^{f_{i}}$.

3. Пусть $f_{i}=\left\lfloor F_{i} / 2\right\rfloor$ и $2 \nmid F_{i}=1$. Тогда снова $f_{i}^{\prime}=f_{i}$, так что оценка снизу для $N_{0}$ сразу вытекает из рассуждений пункта 1 . Оценка сверху вытекает из того, что число значений $a \in E^{\prime}$ по простому модулю $p_{i}$ не превосходит числа корней многочлена $\varphi$ по модулю $p_{i}$, которое в свою очередь не превосходит 2 , так как не все коэффициенты $\varphi$ делятся на $p_{i}$ (многочлен $\varphi$ является $W$-примитивным).

4. Пусть $f_{i}=\left\lfloor F_{i} / 2\right\rfloor, 2 \nmid F_{i}>1$ и число $a_{0}$ не удовлетворяет расширенной системе $\Sigma_{i}^{\prime}$. Тогда снова $f_{i}^{\prime}=f_{i}$, так что оценка снизу для $N_{0}$ сразу вытекает из рассуждений пункта 1. Оценка сверху вытекает из следующего замечания, которое имеет место в силу формулы Тейлора. Для того чтобы наименьший неотрицательный вычет числа $a_{0}+p_{i}^{f_{i}} t$ по модулю $W$ принадлежал $E^{\prime}$, необходимо, чтобы выполнялось сравнение

$$
\frac{\varphi\left(a_{0}\right)}{p_{i}^{2 f_{i}}}+\frac{\varphi^{\prime}\left(a_{0}\right)}{p_{i}^{f_{i}}} t+\frac{\varphi^{\prime \prime}\left(a_{0}\right)}{2} t^{2} \equiv 0 \quad\left(\bmod p_{i}\right) .
$$


Число $a_{0}$ не удовлетворяет расширенной системе $\Sigma_{i}^{\prime}$, поэтому не все коэффициенты этого многочлена от $t$ делятся на $p_{i}$, следовательно, он имеет не более 2 корней по модулю $p_{i}$, а число вычетов по модулю $p_{i}^{F_{i}}$ элементов $a$ из $E^{\prime}$ не превосходит

$$
2 p_{i}^{F_{i}} / p_{i}^{f_{i}+1}=2 p_{i}^{f_{i}}
$$

5. Пусть $f_{i}=\left\lfloor F_{i} / 2\right\rfloor, 2 \nmid F_{i}>1$ и число $a_{0}$ удовлетворяет расширенной системе $\Sigma_{i}^{\prime}$. Рассмотрим систему наименьших неотрицательных вычетов по модулю $W$ чисел $a=a_{0}+p_{i}^{f_{i}} t$, где $t$ пробегает систему чисел, сравнимых с 0 по модулю $W_{i}^{\prime}$ и сравнимых соответственно с числами $0,1, \ldots, p_{i}^{f_{i}+1}-1$ по модулю $p_{i}^{F_{i}}$. Число таких вычетов равно точно $p_{i}^{f_{i}^{\prime}}=p_{i}^{f_{i}+1}$ и все они принадлежат множеству $E^{\prime}$, так как число $a_{0}$ удовлетворяет расширенной системе $\Sigma_{i}^{\prime}$. Отсюда вытекает оценка снизу для $N_{0}$. Оценка сверху вытекает из рассуждений, аналогичных приведенным в п. 2.

Для доказательства теоремы 6 нам надо показать, что

$$
|E(R, W)| \leqslant W^{O(1 / \ln \ln W)} \max \left(R^{4}, W^{12 / 13} R^{2 / 13}\right)
$$

при любом достаточно большом числе $W$ и любом $R, 1 \leqslant R \leqslant W^{1 / 4}$. Для этого мы разобьем множество $E(R, W)$ на четыре (вообще говоря, пересекающихся) подмножества

$$
E(R, W)=\bigcup_{j=1}^{4} E_{j}(R, W)
$$

и покажем, что приведенная только что оценка выполняется для каждого из них, то есть, что

$$
\left|E_{j}(R, W)\right| \leqslant W^{O(1 / \ln \ln W)} \max \left(R^{4}, W^{12 / 13} R^{2 / 13}\right), \quad j=1, \ldots, 4 .
$$

Этого будет достаточно для доказательства теоремы 6.

Описание и исследование множеств $E_{j}(R, W)$ дается в следующих четырех разделах.

\section{5. Множество $E_{1}(R, W)$}

Пусть $T \in \mathbf{R}_{>0}$ - параметр, который мы определим позже. Зададим множество $E_{1}(R, W)$ как совокупность тех элементов $a \in E(R, W)$, для которых хотя бы в одном из наборов $\left(a_{0}, w, w^{\prime}, v, \varphi\right) \in \eta(a)$ имеет место неравенство $w w^{\prime} \leqslant T^{2}$.

Лемма 7. Справедлива оченка

$$
\left|E_{1}(R, W)\right| \leqslant W^{O(1 / \ln \ln W)}\left(R^{4}+T^{2} R^{2}\right)
$$

Доказательство. Для наборов $\left(a_{0}, w, w^{\prime}, v, \varphi\right) \in \eta(a)$ при $a \in E(R, W)$ справедлива система $\Sigma^{\prime}$ :

$$
\begin{aligned}
\varphi\left(a_{0}\right)=\varphi_{3} a_{0}^{3}+\varphi_{2} a_{0}^{2}+\varphi_{1} a_{0}+\varphi_{0}=0 & \left(\bmod w w^{\prime}\right) \\
\varphi^{\prime}\left(a_{0}\right)=3 \varphi_{3} a_{0}^{2}+2 \varphi_{2} a_{0}+\varphi_{1}=0 & \left(\bmod w^{\prime}\right) \\
\frac{1}{2} \varphi^{\prime \prime}\left(a_{0}\right)=3 \varphi_{3} a_{0}+\varphi_{2}=0 & (\bmod v)
\end{aligned}
$$


Пусть $N_{0}=N_{0}\left(a_{0}, w, w^{\prime}\right)$ - число ненулевых многочленов $\varphi$ с целыми коэффициентами степени, не большей 3 , и высоты, не большей $R$, удовлетворяющих такой системе при условии, что числа $a_{0}, w$ и $w^{\prime}$ фиксированы и $w w^{\prime} \leqslant T^{2}$. Тогда количество элементов множества $E_{1}(R, W)$ не превосходит максимума величины

$$
N=d^{2}(W) w N_{0}\left(3^{\omega(W)} w^{\prime}\right),
$$

взятого по всем $a_{0}, w$ и $w^{\prime}$ с условием $w w^{\prime} \leqslant T^{2}$. Первый множитель в произведении (8) есть оценка сверху для количества всевозможных пар $\left(w, w^{\prime}\right)$, потому что числа $w$ и $w^{\prime}$ делят $W$. Второй множитель дает оценку сверху для количества чисел $a_{0}$ при фиксированных $w$ и $w^{\prime}$. Третий множитель есть число многочленов $\varphi$ для фиксированного набора $a_{0}, w, w^{\prime}$. Последний множитель есть согласно лемме 6 оценка сверху для числа элементов в прообразе $\eta^{-1}\left(a_{0}, w, w^{\prime}, v, \varphi\right)$ при условии, что он не пуст.

Для оценки числа $N$ нам надо оценить $N_{0}$. Для этого рассмотрим два случая.

1. Пусть максимум величины $N$ достигается на наборе $a_{0}, w, w^{\prime}$, в котором $w \leqslant R$. Тогда $v \leqslant R$, потому что всегда $v \mid w$. Количество возможных значений $\varphi_{3}$ не превосходит $2 R+1 \leqslant 3 R$. Если фиксировать одно из них, то для $\varphi_{2}$ из третьего равенства системы $\Sigma^{\prime}$ получим ограничение вида $\varphi_{2}=\varphi_{2.0}(\bmod v)$ с известным $\varphi_{2,0}$, следовательно, количество возможных значений $\varphi_{2}$ при фиксированном $\varphi_{3}$ не превосходит $2 R / v+1 \leqslant 3 R / v$. Аналогичным образом, количество возможных значений $\varphi_{1}$ при фиксированных $\varphi_{3}, \varphi_{2}$ не превосходит $2 R / w+1 \leqslant 3 R / w$ и такая же оценка будет иметь место для количества возможных значений $\varphi_{0}$ при фиксированных $\varphi_{3}, \varphi_{2}$ и $\varphi_{1}$. Подводя итог, получим, что

$$
\begin{aligned}
N_{0} & \leqslant 3 R \frac{3 R}{v} \frac{3 R}{w} \frac{3 R}{w}=\frac{(3 R)^{4}}{w w^{\prime}}, \\
N & \leqslant d^{2}(W) w \frac{(3 R)^{4}}{w w^{\prime}}\left(3^{\omega(W)} w^{\prime}\right)=W^{O(1 / \ln \ln W)} R^{4},
\end{aligned}
$$

и лемма в этом случае доказана.

2. Пусть максимум величины $N$ достигается на наборе $a_{0}, w, w^{\prime}$, в котором $w>R$. Тогда из тех же соображений, что и в п. 1 , для числа $N_{0}$ справедлива оценка

$$
N_{0} \leqslant(3 R)(3 R) 3 \cdot 3=3^{4} R^{2},
$$

поэтому

$$
N \leqslant d^{2}(W) w\left(3^{4} R^{2}\right)\left(3^{\omega(W)} w^{\prime}\right)=W^{O(1 / \ln \ln W)} T^{2} R^{2},
$$

и доказательство леммы закончено.

\section{6. Множество $E_{2}(R, W)$}

При доказательстве теоремы 6 мы будем неоднократно использовать следующее утверждение.

Лемма 8 (лемма 2.7 в [5]). Пусть $P(z) \in \mathrm{C}[z], n=\operatorname{deg} P, P(z)=P_{1}(z) \ldots P_{m}(z)$, $P_{1}(z), \ldots, P_{m}(z) \in \mathrm{C}[z]$. Тогда

$$
2^{-n} L\left(P_{1}\right) \ldots L\left(P_{m}\right) \leqslant L(P) \leqslant L\left(P_{1}\right) \ldots L\left(P_{m}\right),
$$

где через $L(P)$ обозначена сумма модулей коэффичиентов многочлена $P$. 
Всюду ниже через $D_{\varphi}$ обозначается дискриминант многочлена $\varphi$.

Зададим множество $E_{2}(R, W)$ в виде

$$
E_{2}(R, W)=E_{2,1}(R, W) \cup E_{2,2}(R, W),
$$

где $E_{2,1}(R, W)$ - совокупность тех элементов $a \in E(R, W)$, для которых хотя бы в одном из наборов $\left(a_{0}, w, w^{\prime}, v, \varphi\right) \in \eta(a)$ имеет место равенство $\operatorname{deg} \varphi=1$, а $E_{2,2}(R, W)-$ совокупность тех элементов $a \in E(R, W)$, для которых хотя бы в одном из наборов $\left(a_{0}, w, w^{\prime}, v, \varphi\right) \in \eta(a)$ выполняются условия $\operatorname{deg} \varphi \geqslant 2$ и $D_{\varphi}=0$.

Лемма 9. Справедлива оченка

$$
\left|E_{2}(R, W)\right| \leqslant W^{O(1 / \ln \ln W)} W^{2 / 3} R .
$$

Доказательство. Так как любой $W$-примитивный многочлен $\varphi$ степени 1 имеет точно один корень по модулю $W$, количество элементов множества $E_{2,1}(R, W)$ совпадает с количеством допустимых многочленов $\varphi$ степени 1 , то есть не больше $(3 R)^{2}$. Поскольку $R^{2}<W^{2 / 3} R$, число элементов множества $E_{2,1}(R, W)$ удовлетворяет оценке из условия леммы.

Элементы множества $E_{2,2}(R, W)$ являются в $\mathbf{Z} / W \mathbf{Z}$ корнями $W$-примитивных многочленов $\varphi$ степени 2 или 3 , для которых $D_{\varphi}=0$. Каждый такой многочлен разлагается над кольцом $\mathbf{Z}$ на линейные множители:

$$
\varphi(x)=\psi^{2}(x) \chi(x), \quad \psi(x), \chi(x) \in \mathbf{Z}[x], \quad \operatorname{deg} \psi=1, \quad \operatorname{deg} \chi \leqslant 1,
$$

которые ввиду его $W$-примитивности будут также $W$-примитивны.

Определим для каждого многочлена $\varphi$ натуральные числа $W_{1}$ и $W_{2}$ с помощью следующих двух формул:

$$
\begin{aligned}
& W_{1}=\max \left\{W^{\prime}: W^{\prime} \mid W, \psi(a)=0 \quad\left(\bmod W^{\prime}\right)\right\}, \\
& W_{2}=\max \left\{W^{\prime}: W^{\prime} \mid W, \chi(a)=0 \quad\left(\bmod W^{\prime}\right)\right\} .
\end{aligned}
$$

Тогда, поскольку $\varphi(a)=0(\bmod W)$, то $W_{1}^{2} W_{2} \geqslant W$.

Пусть

$$
\begin{aligned}
\psi(x) & =\psi_{1} x+\psi_{0}, & \chi(x) & =\chi_{1} x+\chi_{0}, \\
R_{\psi} & =\max \left(\left|\psi_{1}\right|,\left|\psi_{0}\right|\right), & R_{\chi} & =\max \left(\left|\chi_{1}\right|,\left|\chi_{0}\right|\right) .
\end{aligned}
$$

Тогда, пользуясь леммой 8 , находим, что $2^{-3} R_{\psi}^{2} R_{\chi} \leqslant 4 R$, то есть $R_{\psi}^{2} R_{\chi} \leqslant 2^{5} R$.

Множество $E_{2,2}(R, W)$ можно представить в виде объединения подмножества $E_{2,2}^{(1)}(R, W)$, совокупности чисел $a \in E_{2,2}(R, W)$, для которых по крайней мере на одном из элементов множества $\eta(a)$ имеет место неравенство $W_{1} \geqslant W^{3 / 8}$, и подмножества $E_{2,2}^{(2)}(R, W)$, совокупности чисел $a \in E_{2,2}(R, W)$, для которых по крайней мере на одном из элементов множества $\eta(a)$ имеет место неравенство $W_{1}<W^{3 / 8}$.

Заметим, что одно и то же число а может, вообще говоря, иметь несколько наборов $\left(a_{0}, w, w^{\prime}, v, \varphi\right) \in \eta(a)$, для которых выполнены условия $\operatorname{deg} \varphi \geqslant 2$ и $D_{\varphi}=0$, поэтому пересечение указанных подмножеств может быть непустым. Это обстоятельство, как и раньше, не имеет значения. 
Для доказательства требуемой верхней оценки величины $\left|E_{2}(R, W)\right|$ достаточно установить аналогичную оценку для $\left|E_{2,2}(R, W)\right|$, что в свою очередь сводится к доказательству такой же оценки в случае множеств $E_{2,2}^{(j)}(R, W), j=1,2$.

Пусть $a \in E_{2,2}^{(1)}(R, W)$. При фиксированном многочлене $\psi$ число $a$ будет однозначно определено по мсдулю $W_{1}$, потому что многочлен $\psi$ является $W$-примитивным. Следовательно, число возможных значений $a$ по модулю $W$ при фиксированном $\psi$ не превосходит $W / W_{1}$, а так как число допустимых многочленов $\psi$ не превосходит $\left(3 R_{\psi}\right)^{2} \leqslant 3^{2} 2^{5} R$, общее число элементов множества $E_{2,2}^{(1)}(R, W)$ не превосходит

$$
\left(W / W_{1}\right)\left(3^{2} 2^{5} R\right) \leqslant 3^{2} 2^{5} W^{5 / 8} R \leqslant W^{2 / 3} R,
$$

и доказательство в этом случае завершается.

Пусть $a \in E_{2,2}^{(2)}(R, W)$. Положим

$$
W_{1}^{\prime}=\min \left(W_{1}, W_{2}\right), \quad W_{2}^{\prime}=\max \left(W_{1}, W_{2}\right) .
$$

При фиксированных многочленах $\psi$ и $\chi$ число $a$ будет однозначно определено по модулю $W_{2}^{\prime}$, потому что многочлены $\psi$ и $\chi$ являются $W$-примитивными. Следовательно, число возможных значений $a$ по модулю $W$ при фиксированных $\psi$ и $\chi$ не превосходит $W / W_{2}^{\prime}$. Оценим число допустимых пар $(\psi, \chi)$. Поскольку имеют место сравнения

$$
\psi_{1} a+\psi_{0}=0 \quad\left(\bmod W_{1}^{\prime}\right), \quad \chi_{1} a+\chi_{0}=0 \quad\left(\bmod W_{1}^{\prime}\right),
$$

то $\psi_{1} \chi_{0}-\chi_{1} \psi_{0}=0\left(\bmod W_{1}^{\prime}\right)$. Таким образом, $\psi_{1} \chi_{0}-\chi_{1} \psi_{0}=k W_{1}^{\prime}$, причем общее число возможных значений $k$ не превосходит

$$
\frac{2\left(R_{\chi} R_{\psi}+R_{\psi} R_{\chi}\right)}{W_{1}^{\prime}}+1=\frac{4 R_{\psi} R_{\chi}}{W_{1}^{\prime}}+1 .
$$

Для оценки числа допустимых пар $(\psi, \chi)$ и, соответственно, числа элементов множества $E_{2,2}^{(2)}(R, W)$ нам снова потребуется разбиение на подмножества. Пусть $E_{2,2}^{(2,1)}(R, W)$ - совокупность тех чисел $a \in E_{2,2}^{(2)}(R, W)$, для которых по крайней мере на одном из элементов множества $\eta(a)$ выполнено условие $\chi_{0} \neq 0, E_{2,2}^{(2,2)}(R, W)-$ совокупность тех чисел $a \in E_{2,2}^{(2)}(R, W)$, для которых по крайней мере на одном из элементов множества $\eta(a)$ выполнены условия $\chi_{0}=0, \psi_{0} \neq 0, E_{2,2}^{(2,3)}(R, W)-$ совокупность тех чисел $a \in E_{2,2}^{(2)}(R, W)$, для которых по крайней мере на одном из элементов множества $\eta(a)$ выполнены условия $\chi_{0}=0, \psi_{0}=0$.

Рассмотрим первый случай, когда $\chi_{0} \neq 0$. Если зафиксировать набор $\left(k, \chi_{1}, \psi_{0}\right)$, то произведение $\psi_{1} \chi_{0}$ будет иметь фиксированное ненулевое значение $S$, по модулю не превосходящее

$$
R_{\psi} R_{\chi} \leqslant 2^{5} R \leqslant 2^{5} W^{1 / 4} \leqslant W,
$$

поэтому число возможных значений пары $\left(\psi_{1}, \chi_{0}\right)$ для фиксированного набора $\left(k, \chi_{1}, \psi_{0}\right)$ не превосходит

$$
2 \max _{1 \leqslant S \leqslant W} d(S)
$$


а общее число возможных наборов $\left(k, \chi_{1}, \psi_{0}, \psi_{1}, \chi_{0}\right)$ не превосходит

$$
\begin{aligned}
& \left(\frac{4 R_{\psi} R_{\chi}}{W_{1}^{\prime}}+1\right)\left(3 R_{\chi}\right)\left(3 R_{\psi}\right)\left(2 \max _{1 \leqslant S \leqslant W} d(S)\right) \\
& \leqslant \frac{2^{2} 3^{2} 2^{5} R R_{\chi}}{W_{1}^{\prime}} 2 \max _{1 \leqslant S \leqslant W} d(S)+3^{2} 2^{5} R 2 \max _{1 \leqslant S \leqslant W} d(S) \\
& \leqslant\left(\frac{2^{12} 3^{2} R^{2}}{W_{1}^{\prime}}+2^{5} 3^{2} R\right) 2 \max _{1 \leqslant S \leqslant W} d(S) \leqslant\left(\frac{R^{2}}{W_{1}^{\prime}}+R\right) W^{O(1 / \ln \ln W) .}
\end{aligned}
$$

Так как $W_{1}^{2} W_{2} \geqslant W$, то $W_{1}^{\prime} W_{2}^{\prime}=W_{1} W_{2} \geqslant\left(W / W_{1}\right)$ и $W_{2}^{\prime} \geqslant W^{1 / 3}$, поэтому общее число элементов множества $E_{2,2}^{(2,1)}(R, W)$ не будет превосходить

$$
\begin{aligned}
& \frac{W}{W_{2}^{\prime}}\left(\frac{R^{2}}{W_{1}^{\prime}}+R\right) W^{O(1 / \ln \ln W)} \leqslant\left(\frac{W R^{2}}{W / W_{1}}+\frac{W R}{W^{1 / 3}}\right) W^{O(1 / \ln \ln W)} \\
& =\left(W_{1} R^{2}+W^{2 / 3} R\right) W^{O(1 / \ln \ln W)} \leqslant\left(W^{3 / 8} R^{2}+W^{2 / 3} R\right) W^{O(1 / \ln \ln W)} \\
& \leqslant\left(W^{3 / 8+1 / 4} R+W^{2 / 3} R\right) W^{O(1 / \ln \ln W)} \leqslant W^{2 / 3} R W^{O(1 / \ln \ln W)}
\end{aligned}
$$

и доказательство в этом случае заканчивается.

Перейдем ко второму случаю, когда $\chi_{0}=0, \psi_{0} \neq 0$. Тогда $\chi_{1} \neq 0$. Если зафиксировать набор $\left(k, \chi_{0}, \psi_{1}\right)$, то произведение $\psi_{0} \chi_{1}$ будет иметь фиксированное ненулевое значение $S$, по модулю не превосходящее (10), поэтому число возможных значений пары $\left(\psi_{0}, \chi_{1}\right)$ для фиксированного набора $\left(k, \chi_{0}, \psi_{1}\right)$ не превосходит величины (11). Общее число возможных наборов $\left(k, \chi_{0}, \psi_{1}, \psi_{0}, \chi_{1}\right)$ можно снова оценить величиной $(12)$, а общее число рассматриваемых значений $a-$ величиной (13).

Пусть, наконец, $\chi_{0}=0, \psi_{0}=0$. Тогда количество допустимых пар $(\psi, \chi)$ не превосходит

$$
\left(3 R_{\psi}\right)\left(3 R_{\chi}\right) \leqslant 3^{2}\left(2^{5} R\right),
$$

а общее число элементов множества $E_{2,2}^{(2,3)}(R, W)$ не превосходит

$$
\frac{W}{W_{2}^{\prime}}\left(2^{5} 3^{2} R\right) \leqslant 2^{5} 3^{2} W^{2 / 3} R \text {, }
$$

чем и заканчивается доказательство леммы.

\section{7. Множество $E_{3}(R, W)$}

Для фиксированного набора $\left(a_{0}, w w^{\prime}, w^{\prime}, v\right)$ рассмотрим решетку

$$
\begin{aligned}
\mathscr{L}_{3}\left(a_{0}\right) & =\mathscr{L}_{3}\left(a_{0}, w w^{\prime}, w^{\prime}, v\right) \\
& =\left\{\left(\varphi_{0}, \varphi_{1}, \varphi_{2}, \varphi_{3}\right) \in \mathbf{Z}^{4}: \text { многочлен } \varphi(x)=\sum_{j=0}^{3} \varphi_{j} x^{j} \text { удовлетворяет системе } \Sigma^{\prime}\right\},
\end{aligned}
$$

где $\Sigma^{\prime}-$ система из доказательства леммы 7. 
Пусть $E_{3}(R, W)$ - совокупность тех элементов $a \in E(R, W)$, для которых хотя бы в одном из наборов $\left(a_{0}, w, w^{\prime}, v, \varphi\right) \in \eta(a)$ имеют место неравенства

$$
w w^{\prime}>T^{2}, \quad \lambda_{1}\left(\mathscr{L}_{3}\left(a_{0}\right)\right) \leqslant \frac{\left(w w^{\prime}\right)^{1 / 4}}{3^{r / 2+1}},
$$

где $\lambda_{1}(\cdot)-$ первый последовательный минимум Минковского.

Лемма 10. Справедлива оченка

$$
\left|E_{3}(R, W)\right| \leqslant W^{O(1 / \ln \ln W)} W / T^{1 / 6} .
$$

Доказательство. План доказательства леммы будет таким. Пусть $a \in E_{3}(R, W)$ (то есть выполнены условия (14)), и пусть $\varphi^{*}(x)$ - многочлен, на котором достигается последовательный минимум $\lambda_{1}=\lambda_{1}\left(\mathscr{L}_{3}\left(a_{0}\right)\right)$. Сначала мы покажем, что указанная в (14) граница для числа $\lambda_{1}$ влечет выполнение по крайней мере одного из равенств $\operatorname{deg} \varphi^{*}=1, D_{\varphi^{*}}=0$, а затем установим требуемую оценку количества элементов множества $E_{3}(R, W)$ приблизительно тем же способом, что и при доказательстве леммы 9.

Допустим противное, то есть, что $\operatorname{deg} \varphi^{*} \geqslant 2$ и $D_{\varphi^{*}} \neq 0$. Тогда числа $\rho_{i}=v_{p_{i}}\left(D_{\varphi^{*}}\right)$ конечны. По лемме 5 число корней многочлена $\varphi^{*}$ по модулю $p_{i}^{f_{i}+f_{i}^{\prime}}$ не превосходит $3 p_{i}^{\rho_{i} / 2}$, отсюда по китайской теореме об остатках число корней многочлена $\varphi^{*}$ по модулю $w w^{\prime}$ не превосходит $3^{r} \sqrt{D_{\varphi^{*}}}$. Заметим, что дискриминант многочлена общего вида второй степени $A_{2} x^{2}+A_{1} x+A_{0}$ есть однородный многочлен $A_{1}^{2}-4 A_{0} A_{2}$ степени 2 от букв $A_{2}, A_{1}, A_{0}$, сумма модулей коэффициентов которого не превосходит 5 , а дискриминант многочлена общего вида третьей степени $A_{3} x^{3}+A_{2} x^{2}+A_{1} x+A_{0}$ есть однородный многочлен $-4 A_{1}^{3} A_{3}-4 A_{2}^{3} A_{0}+A_{1}^{2} A_{2}^{2}-27 A_{0}^{2} A_{3}^{3}+18 A_{0} A_{1} A_{2} A_{3}$ степени 4 от букв $A_{3}$, $A_{2}, A_{1}, A_{0}$, сумма модулей коэффициентов которого не превосходит 54. Следовательно, если $R_{\varphi^{*}}$ - максимум модулей коэффициентов $\varphi^{*}$, то число корней $\varphi^{*}$ по модулю $w w^{\prime}$ не превосходит

$$
3^{r} \sqrt{D_{\varphi^{*}}} \leqslant 3^{r} \sqrt{54 R_{\varphi^{*}}^{4}} \leqslant 3^{r} \sqrt{54} \lambda_{1}^{2} \leqslant 3^{r} \sqrt{54}\left(\frac{1}{3^{r / 2+1}}\left(w w^{\prime}\right)^{1 / 4}\right)^{2}<\left(w w^{\prime}\right)^{1 / 2} \leqslant w^{\prime} .
$$

С другой стороны, из формулы Тейлора и из того, что набор коэффициентов многочлена $\varphi^{*}$ принадлежит решетке $\mathscr{L}_{3}\left(a_{0}\right)$, вытекает, что $\varphi^{*}\left(a_{0}+w t\right)=0\left(\bmod w w^{\prime}\right)$ при любом целом $t$. Взяв, в частности, $t=0,1, \ldots, w^{\prime}-1$, получим $w^{\prime}$ корней по модулю $w w^{\prime}$, что противоречит предыдущей оценке. Значит, $D_{\varphi^{*}}=0$.

Далее мы действуем по той же схеме, что и при доказательстве леммы 9. Основное отличие на этот раз в том, что многочлен $\varphi^{*}$ может быть не $W$-примитивен. Пусть $\xi-$ натуральное число, делящее $W$ и такое, что многочлен $\varphi^{*} / \xi$ является $W$-примитивным, а $W^{*}-$ наибольшее натуральное число, делящее $W$ и такое, что $\varphi^{*}(a)=0\left(\bmod W^{*}\right)$. Мы уже видели, что $\varphi^{*}\left(a_{0}+w t\right)=0\left(\bmod w w^{\prime}\right)$ для целых $t$. Так как $a=a_{0}(\bmod w)$, то $\varphi^{*}(a)=0\left(\bmod w w^{\prime}\right)$ и $W^{*} \geqslant w w^{\prime} . \mathrm{B}$ случае $\operatorname{deg} \varphi^{*}=1$ (аналог множества $E_{2,1}(R, W)$ леммы 9) мы получим, что при фиксированных $\xi$ и $\varphi^{*} / \xi$ число а будет определено однозначно по модулю $W^{*} / \xi$. Число допустимых $W$-примитивных многочленов $\varphi^{*} / \xi$ не превосходит $\left(3 \lambda_{1} / \xi\right)^{2}$, поэтому для числа $N$ рассматриваемых значений $a$ справедлива оценка

$$
\begin{aligned}
N & \leqslant \sum_{\xi \mid W} \frac{W}{W^{*} / \xi}\left(3 \lambda_{1} / \xi\right)^{2} \leqslant \frac{W}{W^{*}} \frac{\left(w w^{\prime}\right)^{1 / 2}}{3^{\omega(W)}} d(W) \\
& \leqslant W^{O(1 / \ln \ln W)} W /\left(w w^{\prime}\right)^{1 / 2} \leqslant W^{O(1 / \ln \ln W)} W / T,
\end{aligned}
$$


завершающая в данном случае доказательство леммы.

В случае $\operatorname{deg} \varphi^{*} \geqslant 2, D_{\varphi^{*}}=0$ (аналог множества $E_{2,2}(R, W)$ леммы 9) многочлен $\varphi^{*}$ будет удовлетворять равенству вида

$$
\varphi^{*}(x)=\xi \psi^{2}(x) \chi(x), \quad \psi(x), \chi(x) \in \mathbf{Z}[x], \quad \operatorname{deg} \psi=1, \quad \operatorname{deg} \chi \leqslant 1 .
$$

Так как $\varphi^{*} / \xi$ является $W$-примитивным, то $\psi$ и $\chi$ также $W$-примитивны.

Пусть величины $W_{1}, W_{2}, \psi_{1}, \psi_{0}, \chi_{1}, \chi_{0}, R_{\psi}, R_{\chi}, W_{1}^{\prime}$ и $W_{2}^{\prime}$ определяются так же, как и в лемме 9. Тогда числа $R_{\psi}$ и $R_{\chi}$ удовлетворяют оценке $R_{\psi}^{2} R_{\chi} \leqslant 2^{5} \lambda_{1} / \xi$. Рассмотрим два случая.

1. Пусть $W_{1} \geqslant W^{* 3 / 8}$ (аналог множества $E_{2.2}^{(1)}(R, W)$ леммы 9). Число допустимых многочленов $\psi$ при фиксированном $\xi$ не превосходит $3^{2} 2^{5} \lambda_{1} / \xi$, а если зафиксировать числа $\xi, \psi_{1}$ и $\psi_{0}$, то число $a$ будет однозначно определено по модулю $W_{1}$. Отсюда для числа $N$ вытекает оценка

$$
\begin{aligned}
N & \leqslant \sum_{\xi \mid W} \frac{W}{W_{1}}\left(3^{2} 2^{5} \lambda_{1} / \xi\right) \leqslant 3^{2} 2^{5} d(W) W \lambda_{1} /\left(W^{*}\right)^{3 / 8} \leqslant W^{O(1 / \ln \ln W)} \frac{W}{\left(w w^{\prime}\right)^{3 / 8}}\left(w w^{\prime}\right)^{1 / 4} \\
& \leqslant W^{O(1 / \ln \ln W)} \frac{W}{\left(w w^{\prime}\right)^{1 / 8}} \leqslant W^{O(1 / \ln \ln W)} W / T^{1 / 4}
\end{aligned}
$$

и лемма в этом случае доказана.

2. Пусть $W_{1}<W^{* 3 / 8}$ (аналог множества $E_{2,2}^{(2)}(R, W)$ леммы 9). При фиксированных многочленах $\psi$ и $\chi$ число а однозначно определяется по модулю $W_{2}^{\prime}$. Числа $\psi_{1}, \psi_{0}, \chi_{1}$, $\chi_{0}$ удовлетворяют равенству $\psi_{1} \chi_{0}-\chi_{1} \psi_{0}=k W_{1}^{\prime}$ с целым $k$. Если зафиксировать $\xi$, то количество возможных значений $k$ не будет превосходить величины (9). Рассмотрим снова три случая.

2.1. Пусть $\chi_{0} \neq 0$ (аналог множества $E_{2,2}^{(2,1)}(R, W)$ леммы 9). Из того, что

$$
R_{\psi} R_{\chi} \leqslant 2^{5} \frac{\lambda_{1}}{\xi} \leqslant 2^{5}\left(w w^{\prime}\right)^{1 / 4} \leqslant 2^{5} W^{1 / 2} \leqslant W
$$

вытекает, что верхняя граница (11) для числа допустимых наборов $\left(\psi_{1}, \chi_{0}\right)$ при фиксированных $k, \chi_{1}, \psi_{0}$ остается в силе. Оценка (12) числа допустимых наборов $\left(k, \chi_{1}, \psi_{0}, \psi_{1}, \chi_{0}\right)$ при фиксированном $\xi$ также останется в силе, если всюду вместо $R$ в ней поставить $\lambda_{1} / \xi$. Наконец, поскольку $W_{1}^{\prime} W_{2}^{\prime} \geqslant W^{*} /\left(\xi W_{1}\right)$ и $W_{2}^{\prime} \geqslant\left(W^{*} / \xi\right)^{1 / 3}$, то аналогом формул (13) для числа $N$ будут формулы

$$
\begin{aligned}
N & \leqslant \sum_{\xi \mid W} \frac{W}{W_{2}^{\prime}}\left(\frac{\left(w w^{\prime}\right)^{1 / 2}}{\xi^{2} W_{1}^{\prime}}+\frac{\left(w w^{\prime}\right)^{1 / 4}}{\xi}\right) W^{O(1 / \ln \ln W)} \\
& \leqslant \sum_{\xi \mid W}\left(\frac{W\left(w w^{\prime}\right)^{1 / 2}}{\xi^{2} W^{*} /\left(\xi W_{1}\right)}+\frac{W\left(w w^{\prime}\right)^{1 / 4}}{\left(W^{*} / \xi\right)^{1 / 3} \xi}\right) W^{O(1 / \ln \ln W)} \\
& \leqslant \sum_{\xi \mid W}\left(\frac{W\left(w w^{\prime}\right)^{1 / 2}}{\xi W^{* 5 / 8}}+\frac{W\left(w w^{\prime}\right)^{1 / 4}}{W^{* 1 / 3} \xi^{2 / 3}}\right) W^{O(1 / \ln \ln W)} \\
& \leqslant d(W)\left(\frac{W\left(w w^{\prime}\right)^{1 / 2}}{\left(w w^{\prime}\right)^{5 / 8}}+\frac{W\left(w w^{\prime}\right)^{1 / 4}}{\left(w w^{\prime}\right)^{1 / 3}}\right) W^{O(1 / \ln \ln W)} \\
& \leqslant \frac{W}{\left(w w^{\prime}\right)^{1 / 12} W^{O(1 / \ln \ln W)} \leqslant W^{O(1 / \ln \ln W)} W / T^{1 / 6}}
\end{aligned}
$$


и лемма в этом случае доказана.

2.2. Если $\chi_{0}=0, \psi_{0} \neq 0$, то из анализа соответствующего случая леммы 9 (множество $\left.E_{2,2}^{(2,2)}(R, W)\right)$ нетрудно заключить, что для числа $N$ рассматриваемых значений $a$ оценка (15) имеет место.

2.3. Пусть, наконец, $\chi_{0}=0, \psi_{0}=0$ (аналог множества $E_{2.2}^{(2.3)}(R, W)$ леммы 9). Тогда при фиксированном $\xi$ число допустимых пар $(\psi, \chi)$ не превосходит $3^{2} 2^{5} \lambda_{1} / \xi$, а для числа $N$ справедлива оценка

$$
\begin{aligned}
N & \leqslant \sum_{\xi \mid W} \frac{W}{W_{2}^{\prime}}\left(3^{2} 2^{5} \lambda_{1} / \xi\right) \leqslant \sum_{\xi \mid W} \frac{W}{\left(W^{*} / \xi\right)^{1 / 3}}\left(3^{2} 2^{5}\left(w w^{\prime}\right)^{1 / 4} / \xi\right) \\
& \leqslant 2^{5} 3^{2} d(W) \frac{W\left(w w^{\prime}\right)^{1 / 4}}{W^{* 1 / 3}} \leqslant W^{O(1 / \ln \ln W)} \frac{W}{\left(w w^{\prime}\right)^{1 / 12}} \leqslant W^{O(1 / \ln \ln W)} W / T^{1 / 6},
\end{aligned}
$$

завершающая доказательство леммы.

Теперь выберем параметр $T$ так, чтобы сбалансировать оценки лемм 7 и 10 , то есть определим $T$ с помощью равенства $T^{2} R^{2}=W / T^{1 / 6}$, что дает $T=\left(W / R^{2}\right)^{6 / 13}$ и

$$
T^{2} R^{2}=W / T^{1 / 6}=\frac{W}{\left(W / R^{2}\right)^{1 / 13}}=W^{12 / 13} R^{2 / 13}
$$

Отсюда с учетом лемм 7 и 10 получаем, что множества $E_{1}(R, W)$ и $E_{3}(R, W)$ удовлетворяют неравенству (7). Множество $E_{2}(R, W)$ также удовлетворяет этому неравенству, поскольку

$$
W^{2 / 3} R \leqslant W^{2 / 3+11 /(4 \cdot 13)} R^{2 / 13}=W^{137 /(12 \cdot 13)} R^{2 / 13}<W^{12 / 13} R^{2 / 13}
$$

В следующем разделе будет рассмотрен последний случай, анализ которого (лемма 11) завершит доказательство теоремы 3.

\section{8. Множество $E_{4}(R, W)$}

Пусть $E_{4}(R, W)$ - совокупность тех элементов $a \in E(R, W)$, для которых хотя бы в одном из наборов $\left(a_{0}, w, w^{\prime}, v, \varphi\right) \in \eta(a)$ выполнены условия

$$
D_{\varphi} \neq 0, \quad \lambda_{1}\left(\mathscr{L}_{3}\left(a_{0}\right)\right)>\frac{\left(w w^{\prime}\right)^{1 / 4}}{3^{r / 2+1}} .
$$

Ясно, что множество $E_{4}(R, W)$ - это именно то, что осталось рассмотреть для доказательства теоремы 6, то есть имеет место равенство (6).

Лемма 11. Справедлива оченка

$$
\left|E_{4}(R, W)\right| \leqslant W^{O(\mathrm{i} / \ln \ln W)} R^{4} .
$$

Доказательство. Повторяя соответствующее рассуждение предыдущей леммы, получим, что число корней многочлена $\varphi$ по модулю $W$ не превосходит

$$
3^{r} \sqrt{D_{\varphi}} \leqslant 3^{r} \sqrt{54} R^{2} \leqslant 3^{r+2} R^{2}
$$


для любого набора $\left(a_{0}, w w^{\prime}, w^{\prime}, v, \varphi\right) \in \eta(a)$. С другой стороны, по лемме 6 число корней $\varphi$ не меныше $w^{\prime}$. Отсюда вытекает, что $w^{\prime} \leqslant 3^{r+2} R^{2}$.

Пусть для фиксированных $a_{0}, w$ и $w^{\prime}$ величина $N_{0}=N_{0}\left(a_{0}, w, w^{\prime}\right)$ обозначает количество ненулевых многочленов $\varphi(x)=\sum_{j=0}^{3} \varphi_{j} x^{j}$ с целыми коэффициентами степени, не большей 3 , и высоты, не большей $R$, для которых вектор $\left(\varphi_{0}, \varphi_{1}, \varphi_{2}, \varphi_{3}\right)$ принадлежит решетке $\mathscr{L}_{3}\left(a_{0}\right)$. Тогда аналогично тому, как это делалось в лемме 7 , несложно показать, что число элементов множества $E_{4}(R, W)$ не превосходит максимума величины

$$
N=d^{2}(W) w N_{0}\left(3^{\omega(W)} w^{\prime}\right)
$$

взятого по всем $a_{0}, w$ и $w^{\prime}$.

Заметим, что мы рассматриваем лишь те числа $a_{0}$, для которых

$$
\lambda_{1}=\lambda_{1}\left(\mathscr{L}_{3}\left(a_{0}\right)\right)>\frac{1}{3^{r / 2+1}}\left(w w^{\prime}\right)^{1 / 4} .
$$

Для оценки величины $N_{0}$ мы используем следующую идею. Пусть $u_{1}, \ldots, u_{N_{0}}-$ все ненулевые точки решетки $\mathscr{L}_{3}\left(a_{0}\right)$, принадлежащие кубу

$$
2(0 ; 2 R)=\left\{\left(x_{0}, x_{1}, x_{2}, x_{3}\right) \in \mathbf{R}^{4}:\left|x_{i}\right| \leqslant R, i=0,1,2,3\right\}
$$

со стороной $2 R$. Тогда шары радиуса $\lambda_{1} / 2$ с центрами в этих точках лежат в кубе $Q\left(0 ; 2 R+\lambda_{1}\right)$ со стороной $2 R+\lambda_{1}$ и пересечение любых двух таких шаров состоит самое большее из одной точки. Следовательно, суммарный объем всех этих шаров не превосходит объема куба $Q\left(0 ; 2 R+\lambda_{1}\right)$. Объем шара в $\mathbf{R}^{4}$ радиуса $\lambda_{1} / 2$ есть $\left(\pi^{2} / 2\right)\left(\lambda_{1} / 2\right)^{4}$, поэтому справедливо следующее неравенство для объемов:

$$
N_{0} \frac{\pi^{2}}{2}\left(\frac{\lambda_{1}}{2}\right)^{4} \leqslant\left(2 R+\lambda_{1}\right)^{4}
$$

отсюда

$$
N_{0} \leqslant \frac{2^{9}}{\pi^{2}}\left(\frac{R}{\lambda_{1}}+\frac{1}{2}\right)^{4} \leqslant \frac{2^{9}}{\pi^{2}}\left(3^{r / 2+1} \frac{R}{\left(w w^{\prime}\right)^{1 / 4}}+\frac{1}{2}\right)^{4} .
$$

Выше мы видели, что $w^{\prime} \leqslant 3^{r+2} R^{2}$, следовательно,

$$
\frac{1}{3^{r / 2+1}}\left(w w^{\prime}\right)^{1 / 4} \leqslant \frac{1}{3^{r / 2+1}}\left(w^{\prime}\right)^{1 / 2} \leqslant R
$$

и

$$
\frac{1}{2} \leqslant \frac{1}{2} 3^{r / 2+1} \frac{R}{\left(w w^{\prime}\right)^{1 / 4}} .
$$

Таким образом, для числа $N_{0}$ получаем неравенство

$$
\begin{aligned}
N_{0} & \leqslant \frac{2^{9}}{\pi^{2}}\left(3^{r / 2+1} \frac{R}{\left(w w^{\prime}\right)^{1 / 4}}+\frac{1}{2} 3^{r / 2+1} \frac{R}{\left(w w^{\prime}\right)^{1 / 4}}\right)^{4} \\
& =\frac{2^{9}}{\pi^{2}} \frac{3^{4}}{2^{4}} 3^{2 r+4} \frac{R^{4}}{w w^{\prime}}=\frac{2^{5} 3^{2 r+8}}{\pi^{2}} \frac{R^{4}}{w w^{\prime}}
\end{aligned}
$$

Подставляя это в (16), получим, что $N \leqslant W^{O(1 / \ln \ln W)} R^{4}$, что и требовалось доказать. 


\section{Список литературы}

1. Боревич З. И., Шафаревич И. Р., Теория чисел. Наука, Москва, 1985.

2. Касселс Дж. В. С., Введепие в геометрию чисел. Мир, Москва, 1965.

3. Коблиц Н., р-адические числа, р-адический алализ и дзета-фуикиии. Бибфизмат, Могилев, 1982.

4. Прахар К., Распределение простых чисел. Мир, Москва, 1967.

5. Фельдман Н. И., Седьмая проблема Гильберта. Изд-во МГУ, Москва, 1982.

6. Frieze A. M., Håstad J., Kannan R., Lagarias J. C., Shamir A., Reconstructing truncated integer variables satisfying linear congruences. SIAM J. Comput. (1988) 17, №2, 262-280.

7. Lagarias J. C., Lenstra H. W., Schnorr C. P., Korkine-Zolotareff bases and successive minima of a lattice and its reciprocal lattice. Tech. Rept., MSRI 07718-86, Math. Sci. Research Inst., Berkeley.

8. Waldschmidt M., Diophantine approximation on linear algebraic groups. Springer, Berlin, 1999.

Статья поступила 10.11.2003. Переработанный вариант поступил 14.09.2004. 